\title{
EVALUATION OF THE DISPERSANTS EFFECTIVENESS USING THE BAFFLED FLASK TEST
}

\author{
Natalia GRECHISHCHEVA ${ }^{1 *}$, Alexandra KUCHIERSKAYA ${ }^{1}$, Anton SEMENOV ${ }^{1}$, \\ Dmitry KURYASHOV ${ }^{2}$, Irakli MERITSIDI ${ }^{1}$, Rifat MINGAZOV ${ }^{2}$ \\ ${ }^{1}$ National University of Oil and Gas "Gubkin University", Leninsky Prospekt 65, \\ 119991 Moscow, Russian Federation \\ ${ }^{2}$ Kazan National Research Technological University, Karl Marx street 68, \\ 420015 Kazan, Republic of Tatarstan, Russian Federation
}

Received 11 August 2020; accepted 17 June 2021

\footnotetext{
Highlights

BFT method does not always provide high accuracy results in the case of heavy oils.

The introduction of heavy oil into the tested systems "by weight" is more preferable.

The introduction of oil into the systems "by weight" minimizes the error of the BFT.

Tested dispersants are less effective on viscous oil.
}

\begin{abstract}
The use of chemical dispersants is one of the most widely used methods for responding to oil spills. The most important characteristic of dispersants is their effectiveness. The dispersant effectiveness is a measure of how well the dispersant breaks up and stabilizes the oil into the water column. In this paper, we studied the dispersing ability of three proprietary dispersants with respect to light and heavy crude oils of Usinsk and Nagornoye fields with a density of $0.816 \mathrm{~g} / \mathrm{cm}^{3}$ and $0.896 \mathrm{~g} / \mathrm{cm}^{3}$, respectively. The dispersant effectiveness was determined using a Baffled Flask Test; dispersant was applied to the oil slick, mixed, and the concentration of oil in a sample taken from the water column was measured using UV-Visible spectrophotometry. A modification of the standard technique is proposed to minimize the error of the method for the heavy crude oil by eliminating the error associated with inaccuracy of dosing. For this purpose, oil of the Nagornoye field was added to the tested systems not "by volume", but "by weight". It was provided better convergence of the experimental results. The standard deviation in the case of dosing of oil "by volume" exceeded $10 \%$ and varied from $11.87 \%$ to $13.59 \%$. The introduction of oil "by weight" was much lower and varied from $5.66 \%$ to $6.30 \%$. Studied dispersants have a higher dispersing ability for the less dense oil of the Usinsk field.
\end{abstract}

Keywords: chemical dispersants, oil spills, Baffled Flask Test, dispersion effectiveness.

\section{Introduction}

The experience of recent years shows that the use of chemical dispersion has become one of the main methods used in the oil spill responses at marine environments (Cai et al., 2017; Lessard \& DeMarco, 2000). This method is based on the use of dispersants, which are compositions of two or more surfactants in various solvents. The stabilizing effect of surfactants in relation to oil dispersions in water is based on a decrease in interfacial tension at the water-oil interface and the formation of a structural mechanical barrier on it as a result of adsorption of surfactant molecules. The decrease in interfacial tension between the oil and water phases contributes to the decomposition of the oil film into separate, smaller drops. In this case, effective dispersion is achieved with a droplet size of 1 to $70 \mu \mathrm{m}$ ( $\mathrm{Li}$ et al., 2011). This process of dispersion of oil in the water column contributes to its biodegradation, as it significantly increases the oil-water contact area and, consequently, the availability of oil for microorganisms-oil degraders. Since the role of dispersant in the spill responses is to intensify the process of dispersing oil in water with further stabilization of the resulting dispersions, its most important characteristic is the dispersing ability, essentially expressing the effectiveness of the dispersant.

*Corresponding author. E-mail: yanat2@mail.ru

Copyright $\odot 2022$ The Author(s). Published by Vilnius Gediminas Technical University

This is an Open Access article distributed under the terms of the Creative Commons Attribution License (http://creativecommons.org/licenses/by/4.0/), which permits unrestricted use, distribution, and reproduction in any medium, provided the original author and source are credited. 
The dispersant effectiveness is a measure of how well the dispersant breaks up and stabilizes the oil into the water column (Panetta et al., 2018). Although in world practice, there is a wide database on the effectiveness of the use of various kinds of dispersants depending on the type of oil, the properties of the dispersant, climatic and other conditions, the question of the optimal method for determining the effectiveness of dispersants remains open. Several methods allow, under given conditions, to evaluate the effectiveness of dispersants using laboratory tests, and however, such studies do not fully allow for the influence of natural factors. In addition, the use of oil with various physical and chemical properties can lead to significant errors in determining the dispersion efficiency. Therefore, an important task is to carry out large-scale experimental work using various oil samples that differ in physical and chemical properties.

To this date, the following methods can be distinguished that are used to determine the effectiveness of dispersants in laboratory conditions:

- French Institute of Petroleum Test (IFP) (Bocard \& Castaing, 1986)

A very satisfactory low energy test simulating field conditions with "non-breaking" waves. The test is very suitable for ranking dispersants. The test is conducted in a test tank with continuous clean seawater inflow at the surface, which causes gradual dilution of the content. To estimate the test method effectiveness, it is crucially important to decrease the mixing energy in order to select the best products i.e., those which remain effective even at low energy level. The method aims to simulate real environmental conditions: contacting dispersant is added onto the oil, and infinite dilution is achieved by the water stream flowing through the test tank. The test tank is a cylindrical glass vessel with two openings: an inlet opening is located just below the water experimental level and an outlet opening is located at the bottom of the vessel. Pure seawater is supplied into a glass vessel through an inlet opening. The test oil is placed in the closed ring and the dispersant is applied on the top. Energy is applied to the test oil onto the surface, by a submerged beater-ring, and dispersed oil is collected from the bottom of the tank. The dispersant effectiveness is measured as the amount of oil that is dispersed and collected from the bottom outflow opening during the test (1 hour).

- Mackay, Nadeau and Steelman Test (MNS) (Mackay et al., 1984)

MNS test is medium energy test simulating field conditions of "breaking waves". The stirring energy is generated by wind - no mechanical energy is supplied, which maximizes the simulation of field conditions. It is less sensitive than IFP-test. The test tank is a closed, thermostatically controlled $6 \mathrm{~L}$ glass vessel, with special openings for supplying oil, dispersant, and air flow. Oil is placed onto the surface of the water using a protective ring, inside which a dispersant is added. The ring prevents oil against spreading over the water surface prior to contact with dispersant. After adding the dispersant, the protective ring is removed and the system continues stirring for 10 minutes. The amount of dispersed oil is determined spectrophotometrically.

- Warren Springs Laboratory Method (WSL) (Lee et al., 1981)

WSL test is a relatively "high energy" test using rotating flasks that make the dispersant-treated oil and water to be mixed completely. This quite simple test is useful for screening dispersants on non-emulsified oils. The test is less representative for field conditions. The test is carried out by adding a premixed mixture of oil and dispersant to a separatory funnel containing a specified amount of water. Then the separating funnel is rotated around the horizontal axis for 2 minutes. After settling, a part of the dispersed oil is taken from the bottom of the funnel to evaluate the effectiveness of dispersant. This method is quite simple and does not require the use of sophisticated equipment.

- Cascading Weir Test (Daling \& Lichtenthaler, 1986)

The test uses a special glass-walled gutter 8 meters long, $20 \mathrm{~cm}$ wide and $7 \mathrm{~cm}$ deep. The gutter is installed at a slight slope, making water to flow down over the barrier to simulate wave action. Water is supplied into the gutter at a constant rate, oil is supplied to the surface of the water and spreads along the entire length of the gutter. The dispersant is sprayed over the oil slick at a predetermined rate. The glass-walled gutter allows the effect of dispersant to be observed on the oil. The method requires sophisticated equipment. In addition the tests require rather large quantities of oil and water, which can cause additional costs associated with disposal.

- Exxon Dispersant Effectiveness Test (ExDET) (Becker et al., 1991)

The ExDET test to estimate the dispersant effectiveness is classified as shake flask testing. This test is similar to WSL test. But in this method, after stirring, a sorbent is placed in the water to collect undispersed oil. The method is also not laborious, but it requires a lot of time compared to the WSL method due to the need to extract oil from the sorbent surface.

- Swirling Flask Test (SFT) (Fingas et al., 1989; National regulations, 1996)

The method consists in using a modified Erlenmeyer flask. Water is added to the flask, after which oil and dispersant are successively added to its surface. Then the flask is shaken for 20 minutes. After settling, a part of the dispersed oil is taken through the side nozzle. At the same time, the integrity of the film formed by undispersed oil on the water surface is not disturbed. The method is quite simple to be carried out, its disadvantages are the "wall effect", as well as the ingress of oil into the sampling nozzle while stirring, which also leads to the fact that this oil does not participate in the dispersion process.

- Baffled Flask Test (BFT) (Venosa et al., 2002)

The method is similar to the Swirling Flask test, but this modification uses a flask with a horizontal outlet, equipped with a sampling valve. It has been found that SFT has provided inferior and poorly reproducible results 
related to the design of the test flask. In this regard, a BFT method has been developed that uses a flask with baffles and gives much better reproducibility and repeatability than a SFT. The advantage of the method is the absence of an experimental error associated with the ingress of undispersed oil into the nozzle. There are also modifications of this method, in which the determination of the amount of oil in samples is carried out using acoustic scattering and laser radiation scattering. These methods also make it possible to determine the size distribution of oil droplets.

In addition to the described methods, there are currently a number of modifications of them, including those that allow carrying out experiments on a larger scale using appropriate units (Brandvik et al., 2013). The literature also describes a method related to testing by shaking, in which, after emulsification in containers, their contents are shock-frozen, followed by separation of the frozen contents and separate determination of the oil content in the middle and bottom block (Salnikov et al., 2017).

After analyzing the data on existing methods for determining of the dispersants effectiveness, we can conclude that methods using shaking flasks, such as WSL, ExDET, SFT, and BFT, are the most cost-effective, simple to use, and do not require any complicated additional equipment. Their additional advantage is the possibility of conducting parallel tests to identify convergence of results, as well as conducting simultaneous tests with various oils or dispersants. Thus, the authors (Trudel et al., 2010) showed that the results obtained using IFP and ExDET techniques in the best way correlated with the data of model studies evaluating the effectiveness of dispersants for oils with viscosities from $18690 \mathrm{mPa} \cdot \mathrm{s}$ to $33400 \mathrm{mPa} \cdot \mathrm{s}$. According to (Holder et al., 2015), the results of model experiments were consistent with those obtained using the BFT laboratory test. However, when studying the dependence of Corexit 9500 dispersant efficiency on oil viscosity, zero dispersant efficiency by the MNS method, was observed at an oil viscosity of 47,000 mPa.s (Fiocco et al., 1999), by the ExDET method was above 20,000 $\mathrm{mPa} \cdot \mathrm{s}$ (Canevari et al., 2001), and in the case of application IFP the dispersant stopped working in the oil viscosity range from $20,000 \mathrm{mPa} \cdot \mathrm{s}$ to $30,000 \mathrm{mPa} \cdot \mathrm{s}$ (Fiocco et al., 1999).

Currently, the most common method for laboratory testing to evaluate the effectiveness of dispersants, ensuring the best convergence of the results, is the BFT method. However, an analysis of the literature shows that in the case of crude oils, it does not always provide high accuracy results (Mukherjee et al., 2011; Panetta et al., 2018; Sorial et al., 2004; Venosa et al., 2002).

The effectiveness of dispersants was evaluated using the SFT and BFT methods by (Sorial et al., 2004). For the tests, 18 dispersing compositions and South Louisiana (SLC) and Prudhoe Bay (PBC) oils were used, which are classified as light and medium by their specific weight by the American Petroleum Institute, respectively. Statistical analysis of the results showed that the coefficient of variation when using the SFT method was $21.9 \%$, while for the BFT it was $7.8 \%$. However, even in the case of BFT, a rather large scatter of the results was observed for individual dispersants. So, when using dispersants Dispersit-SPC 1000 (Polychem, USA), Enersperse 1990 (National Environmental Technology Center, UK) and Shell Dispersant VDC (Environment Canada, Canada), the coefficients of variation (\%) for SLC oil were used 19.4, 28.8, 24.8, and 16.3, 19.9, 17.0 when carrying out experiments by different researchers and one operator, respectively. For PBC oil, the corresponding values were used 29.0, 19.0, 45.3 and 17.2, 11.4, 16.0.

The repeatability and reproducibility of the results of the analysis of the dispersants effectiveness by the BFT method were evaluated (Venosa et al., 2002). In the experiments, oils and dispersants were used the same as in the study (Sorial et al., 2004). For most dispersants, the repeatability and reproducibility factors ranged from 25 to $30 \%$ with a confidence level of $95 \%$. Thus, when testing in one laboratory and testing in different laboratories, the maximum expected discrepancy between the results of two experiments can vary from $\pm 12.5 \%$ to $\pm 15.0 \%$ for a particular dispersant, which is significant.

The effect of oil composition on the dispersion efficiency of Corexit 9500 was studied using the BFT method (Mukherjee et al., 2011). The oils were prepared by the addition of certain components (saturated hydrocarbons, aromatic hydrocarbons, resins, and asphaltenes) previously extracted from Lloyd oil to the stabilized oils Arabian Light and Mars. A standard deviation of more than $10 \%$ was observed during experiments with oils, the final composition of which corresponded to $45 \%$ saturated and $30 \%$ aromatic hydrocarbons, $15 \%$ resins, $10 \%$ asphaltenes (dispersion effectiveness $62.7 \pm 10.6 \%$ ); $45 \%$ saturated and $40 \%$ aromatic hydrocarbons, $5 \%$ resins, $10 \%$ asphaltenes (dispersion effectiveness 64.3 $\pm 12.7 \%$ ).

Similar studies by the BFT method were carried out by (Panetta et al., 2018). The dispersion effectiveness of 10 oils (ANS fresh, Ewing Bank, IFO 120, Alpine, ANS weathered, Anadarko, Doba Chad, Platform Gina fresh, Endicott, Rock) in the presence of Corexit 9500 at $20{ }^{\circ} \mathrm{C}$ exceeded $70 \%$. At the same time, high standard deviations were obtained for crude oil IFO 120 (dispersion effectiveness $84.36 \pm 11.00 \%$ ) and Rock (dispersion effectiveness $74.27 \pm 14.86 \%)$. The authors correlate the results with the high viscosity of the IFO 120 and Rock oil, 1035 cSt, and 2619 cSt, respectively. However, it should be noted that the standard deviations for Platform Gina fresh and Doba Chad oils with higher viscosity of $3.244 \mathrm{cSt}$ and $1.657 \mathrm{cSt}$ were significantly lower and corresponded to $3.35 \%$ and $3.26 \%$.

In connection with the above mentioned, the goal of this work was to determine the dispersant effectiveness for different oils by the BFT method to minimize the error of this method for heavy oil samples. In order to determine the dispersant effectiveness it is proposed to make corrections to procedure for the BFT outlined by (Venosa et al., 
2002) which consists in dosing oil into the system not by "volume", but "by weight".

\section{Materials and methods}

This study used light and heavy crude oils from Usinsk and Nagornoye fields with a density of $0.816 \mathrm{~g} / \mathrm{cm}^{3}$ and $0.896 \mathrm{~g} / \mathrm{cm}^{3}$, respectively, and patented dispersants: sample I (plant food phospholipids), sample II (mixture of alkyl polyglycosides with low toxic anionic surfactants), and sample III (mixture of alkyl sorbitans and anionic surfactants). Physicochemical characteristics of these oils are shown in Table 1.

Table 1. Physicochemical characteristics of the oils

\begin{tabular}{|l|c|c|c|c|}
\hline Oil & $\begin{array}{c}\text { Density at } \\
20^{\circ} \mathrm{C}, \\
\mathrm{g} / \mathrm{cm}^{3}\end{array}$ & $\begin{array}{c}\text { Kinematic } \\
\text { viscosity } \\
\text { at } 20^{\circ} \mathrm{C}, \\
\mathrm{mm}^{2} / \mathrm{s}\end{array}$ & $\begin{array}{c}\text { Pour } \\
\text { point, } \\
{ }^{\circ} \mathrm{C}\end{array}$ & $\begin{array}{c}\text { Type of oil } \\
\text { according to } \\
\text { GOST } \\
\text { R 51858-2002 }\end{array}$ \\
\hline Usinsk & 0.816 & 5.4 & -23 & extra-light \\
\hline Nagornoye & 0.896 & 54 & -14 & heavy \\
\hline
\end{tabular}

During the experiments, dichloromethane (DCM) of qualification "high grade" (Ecos-1, Russia, purity> 99.5\%), freshly prepared distilled water and sodium chloride (Component Reaktiv, Russia, purity> 99.9\%) were used. Following with the BFT procedure (Venosa et al., 2002), standard systems were originally prepared. For this purpose, dichloromethane, oil, and the dispersant under study were added successively into the tank in an amount of 1:20 with respect to oil. At the same time, two standard systems were prepared for each oil, into one of which the oil was introduced "by volume", in accordance with the procedure (Venosa et al., 2002), and into the other by weight, using a Pioneer PA413C laboratory balance (Ohaus, USA), proceeding from the value of the density of oil and the required volume. To evaluate the natural dispersion of oil, similar systems were prepared without the addition of a dispersant. The amount of oil was determined using the calibration method.

To obtain the calibration functions, the following procedure was performed. A certain amount of synthetic seawater (3.5\% wt. $\mathrm{NaCl}$ solution) was added into six separatory funnels and standard systems from 20 to $300 \mu \mathrm{L}$ for Usinsk (light) and 20 to $140 \mu \mathrm{L}$ for Nagornoye (heavy) crude oil were added, respectively. Two series of systems were prepared for each oil ("by volume" and "by weight"). For each calibration sample, the absorption spectrum was measured at a temperature of $22{ }^{\circ} \mathrm{C}$ in the wavelength range of 300-500 nm using a Cary 60 spectrophotometer (Agilent Technologies, USA). The absorbance at three discreet wavelengths of 340,370 , and $400 \mathrm{~nm}$ was recorded, and the area under the absorbance versus wavelength curve was calculated by applying the trapezoidal rule according to the following equation:

$$
\begin{aligned}
& \text { Area }=\left(A b s_{340}+A b s_{370}\right) / 2 \times 30+ \\
& \left(A b s_{370}+A b s_{400}\right) / 2 \times 30
\end{aligned}
$$

where $A b s_{340}, A b s_{370}, A b s_{400}$ is spectrophotometric absorbance at the corresponding wavelength.

This area count was used to calculate the oil dispersed $\left(O_{d}\right)$ and then the dispersion effectiveness of oil.

Evaluation of the effectiveness of dispersants was carried out using a $150-\mathrm{mL}$ trypsinizing flask that has been modified by the placement of a glass stopcock near its bottom so that a subsurface water sample can be removed without disturbing the surface oil layer, as shown in Figure 1.

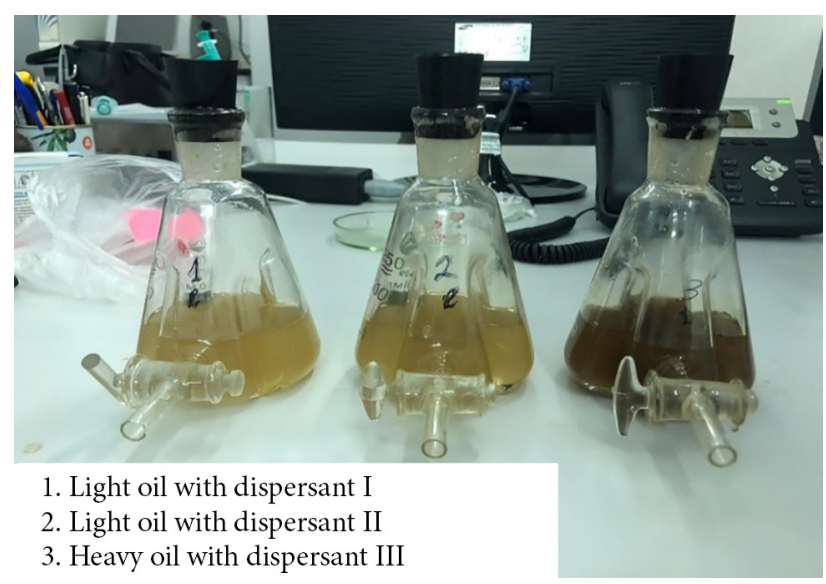

Figure 1. Appearance of modified flasks

Two series of experiments were carried out: in the first of them, oil was dosed "by volume", in the second - "by weight". The dispersant was introduced into the system with respect to oil 1:20, followed by shaking the system on a rotation shaker at $200 \mathrm{rpm}$ for 10 minutes. After shaking, the upper and lower layers were separated, and then the lower layer was extracted with DCM. The obtained extracts were combined; the concentration of dispersed oil in them was determined spectrophotometrically similar to the procedure described for calibration solutions using the previously obtained linear calibration curve functions. If the values of absorbance were too high, the extracts were diluted with DCM.

The amount of oil dispersed $\left(O_{d}\right)$ in the tested systems was determined as follows:

$$
O_{d}=\text { Area } / \operatorname{tg} \alpha \times V_{\mathrm{DCM}} \times V_{\mathrm{H}_{2} \mathrm{O}} / V_{\mathrm{H}_{2} \mathrm{O}}^{*},
$$

where $V_{\mathrm{DCM}}$ - volume of DCM extract, $\mathrm{mL} ; V_{\mathrm{H}_{2} \mathrm{O}}$ - total volume of seawater in flask, $\mathrm{mL} ; V_{\mathrm{H}_{2} \mathrm{O}}^{*}$ - total volume of seawater extracted, $\mathrm{mL}$; $\operatorname{tg} \alpha$ - slope of the calibration curve.

The dispersion effectiveness of oil $\beta, \%$ (without correcting for oil natural dispersion) was estimated as the ratio of oil dispersed in the test system to the total oil added to the system:

$$
\beta=\frac{O_{d}}{\rho_{p} V_{p}} \times 100 \%,
$$

where is $\rho_{p}$ - density of the test oil, $\mathrm{mg} / \mathrm{mL} ; V_{p}$ - volume of oil added to test flask, mL. 
The dispersion efficiency of oil $\left(\beta^{*}, \%\right)$, correcting for natural dispersion, was calculated using Eq. (4):

$$
\beta^{*}=\bar{\beta}-\bar{\beta}_{0},
$$

where $\bar{\beta}$ - average value of the dispersion efficiency of oil, \%; $\bar{\beta}_{0}$ - average percent of oil dispersion without dispersant.

In order to assess the minimum value of the dispersion efficiency determined by this method, the oil dispersion efficiency was carried out taking into account the boundary of the confidence interval (Panetta et al., 2018; Venosa et al., 2002).

Because of this, the final value of the effectiveness of oil dispersion with a probability $(P)$ of $95 \%$ was estimated using the Eq. (5):

$$
\gamma=\beta^{*}-t \times \mathrm{SE}_{\bar{\beta}-\bar{\beta}_{0}},
$$

where $t-95 \%$ critical value for a $\mathrm{t}$-distribution with $\left(n_{\text {disp }}+n_{0}-2\right)$ degrees of freedom; $\mathrm{SE}_{\bar{\beta}-\bar{\beta}_{0}}-$ standard error, defined in Eq. (6):

$$
\mathrm{SE}_{\bar{\beta}-\bar{\beta}_{0}}=\left(s_{\text {disp }}^{2} / n_{\text {disp }}+s_{0}^{2} / n_{0}\right)^{1 / 2} \text {, }
$$

where $s_{\text {disp }}$ - standard deviation of the seven independent replicates $\left(n_{\text {disp }}\right)$ for oil-dispersant systems; $s_{0}$ - standard deviation of the seven independent replicates $\left(n_{0}\right)$ for oil dispersed without dispersant.

a)

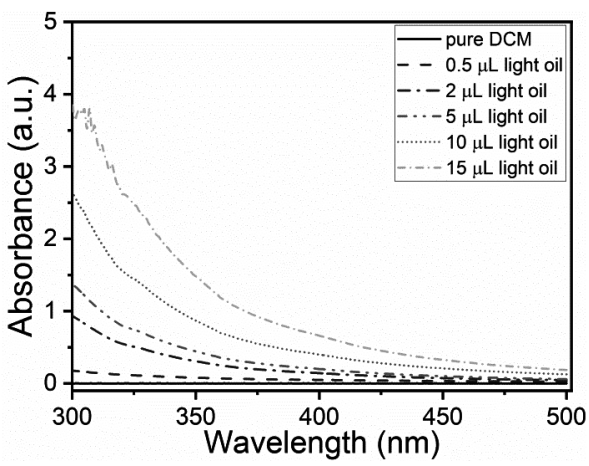

\section{Results and discussion}

Figures 2a, 2b show the absorption spectra of calibration solutions of Usinsk and Nagornoye oils without dispersing agents for dosing oils "by volume". Absorption spectra of calibration solutions of these oils with dispersant I are depicted in Figures 3a, 3b. It can be seen from Figures 2, 3 that an increase in the concentration of Usinsk and $\mathrm{Na}$ gornoye oils in the solution leads to an increase in the absorbance of the dichloromethane solution. Moreover, for pure solvent absorption in the wavelength range of $300 \mathrm{~nm}-500 \mathrm{~nm}$ is completely absent. It follows from the obtained dependences that Nagornoye oil with a higher density at the same concentration absorbs radiation in the near UV and visible range more highly than the lighter Usinsk oil. Figure 4 shows typical calibration curves for determining the content of dispersed oils of Usinsk (Figure $4 \mathrm{a}, 4 \mathrm{~b}$ ) and Nagornoye (Figure 4c, 4d) in the presence and in the absence of dispersant Sample I. Calibration curves are given for cases of introducing oils into the system both "by volume" and "by weight".

From the obtained curves in Figure 4, it follows that there is a linear trend line fit to the area under the calibration curve versus the concentration of both oils in the system (the adjusted determination factor in all cases takes values close to 1 ). As can be seen from the above curves of Figure 4, in the case of Usinsk oil $(\mathrm{a}, \mathrm{b})$, there are no significant differences between the Area values obtained when this oil was introduced into the system "by volume"

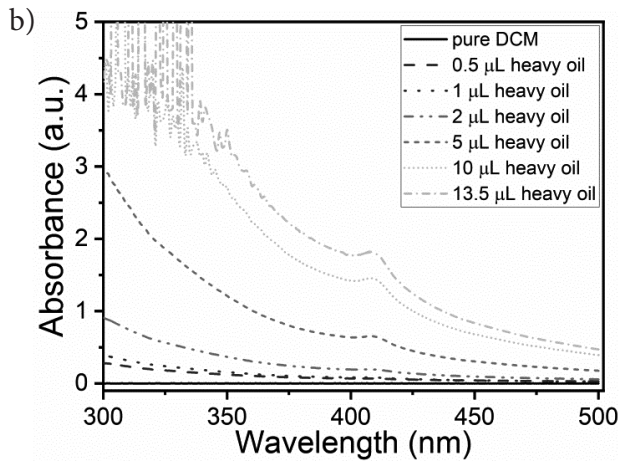

Figure 2. Absorption spectra of calibration solutions of Usinsk (light) oil (a) and Nagornoye (heavy) oil (b) in DCM; the legends show the volume of oil added to $15 \mathrm{~mL}$ of DCM

a)

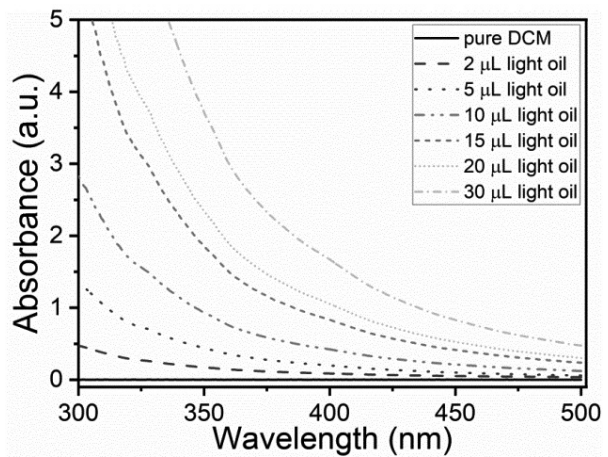

b)

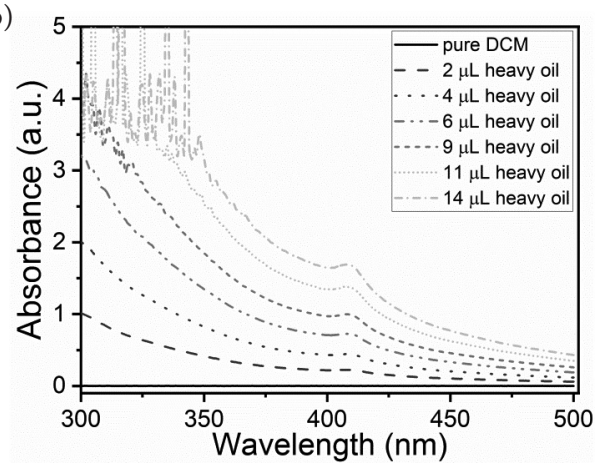

Figure 3. Absorption spectra of calibration solutions of Usinsk (light) oil (a) and Nagornoye (heavy) oil (b) in DCM with dispersant I; the legends show the volume of oil added to $15 \mathrm{~mL}$ of DCM 
a)

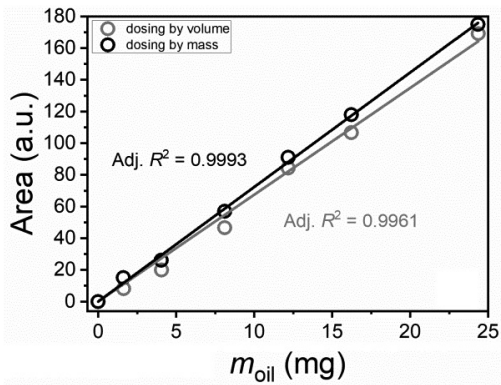

c)

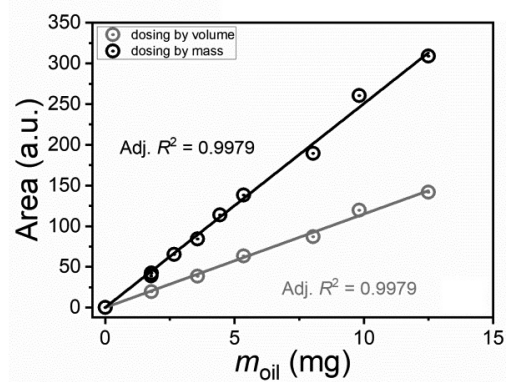

b)

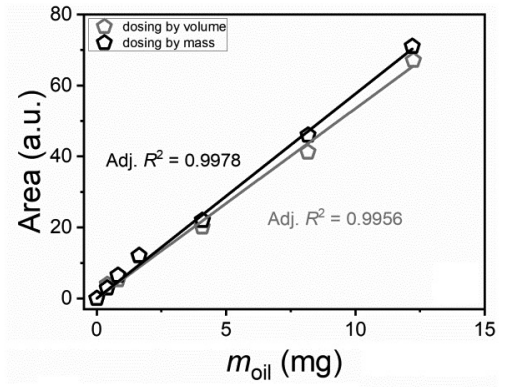

d)

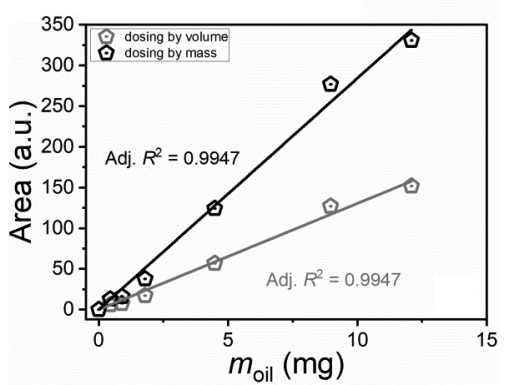

Figure 4. The calibration curves for systems with Usinsk oil with (a) and without (b) dispersant I and Nagornoye oil with (c) and without (d) dispersant I

or "by weight". In the case of Nagornoye oil (Figure 4c, 4d) there is a significant difference in the slopes of the straight lines for cases of adding oil to the system by "volume" and "by weight". This fact is due to the lower actual oil content in the system if it is dosed "by volume" compared to the calculated one. Due to the high viscosity of Nagornoye oil, the introduced volume of this oil does not reflect its final content in the system. In this regard, further experiments to study the degree of dispersion of Usinsk oil were carried out by dosing it "by volume". In the case of Nagornoye oil, the calculations were conducted for two cases: "by volume" and "by weight". Table 2 shows the values of the effectiveness of Usinsk oil dispersion with and without the studied dispersant I-III and the corresponding area of the calibration curves.

As can be seen from the data presented, dispersant III showed the best dispersion of oil, the corresponding $\beta$ exceeded $90 \%$. Moreover, the values of $\beta$ within one sample did not differ from each other in a significant way. The experiments to determine $\beta$ of Nagornoye heavy oil showed a significant scatter of $\beta$ when oil was introduced into the system "by volume". For all dispersants studied the values of $\beta$ varied from $60 \%$ to $140 \%$. This fact, seems, is due to the discrepancy between the actual oil content in the system and the calculated one. Due to the high viscosity of Nagornoye oil, the introduced "by volume" did not reflect the final oil content in the system, which introduced errors in the calculations of $\beta$. To exclude this artifact, oil was added "by weight" to all tested systems. Therewith, in the case of calculating $\beta$ for dosing Nagornoye oil "by volume", values exceeding $100 \%$ were excluded. The average values $\bar{\beta}$ for the tested dispersants are shown in Tables 3 , 4 as well as the values of $\beta^{*}$, calculated taking into account

Table 3. The dispersion effectiveness of Usinsk oil $(n=7)$ at $P=0.95 . \bar{\beta}_{0}=7.60 \%($ Std.Dev. $=0.84)$

\begin{tabular}{|c|c|c|c|c|}
\hline Dispersant & $\bar{\beta}, \%$ & Std.Dev., \% & $\beta^{*}, \%$ & $\gamma, \%$ \\
\hline I & 89.25 & 78.86 & 81.65 & 74.26 \\
\hline II & 90.45 & 9.54 & 82.85 & 77.49 \\
\hline III & 99.92 & 7.34 & 92.93 & 86.59 \\
\hline
\end{tabular}

Table 2. Usinsk oil dispersion effectiveness with $(\beta)$ and without $\left(\beta_{0}\right)$ dispersants I-III and the corresponding area of the calibration curves (Area)

\begin{tabular}{|c|c|c|c|c|c|c|c|c|}
\hline \multirow{2}{*}{ Sample } & \multicolumn{2}{|c|}{ Dispersant I } & \multicolumn{2}{c|}{ Dispersant II } & \multicolumn{2}{c|}{ Dispersant III } & \multicolumn{2}{c|}{ No dispersant } \\
\cline { 2 - 9 } & Area & $\beta, \%$ & Area & $\beta, \%$ & Area & $\beta, \%$ & Area & $\beta_{0}, \%$ \\
\hline 1 & 22.37 & 97.56 & 17.84 & 77.92 & 20.02 & 87.41 & 7.80 & 7.13 \\
\hline 2 & 18.56 & 81.07 & 18.75 & 81.81 & 22.28 & 97.07 & 6.61 & 6.12 \\
\hline 3 & 18.73 & 81.76 & 19.38 & 84.65 & 22.52 & 98.42 & 8.21 & 7.51 \\
\hline 4 & 19.20 & 83.86 & 20.42 & 89.10 & 22.58 & 98.60 & 8.51 & 7.86 \\
\hline 5 & 20.27 & 88.61 & 22.35 & 97.69 & 23.30 & 100.56 & 8.63 & 7.98 \\
\hline 6 & 22.61 & 98.63 & 22.81 & 99.62 & 24.75 & 108.10 & 9.09 & 8.33 \\
\hline 7 & 23.25 & 101.60 & 23.44 & 102.40 & 25.02 & 109.30 & 9.40 & 8.61 \\
\hline
\end{tabular}


Table 4. The dispersion effectiveness of Nagornoye oil $(n=7)$ at $P=0.95 . \bar{\beta}_{0} 4.62($ Std.Dev. $=1.08)$ and $2.30 \%($ Std.Dev. $=0.60)$ when dosing "by volume" and "by weight", respectively

\begin{tabular}{|c|c|c|c|c|c|c|c|c|}
\hline \multirow{2}{*}{ Dispersant } & \multicolumn{2}{|c|}{$\bar{\beta}, \%$} & \multicolumn{2}{c|}{ Std.Dev., \% } & \multicolumn{2}{c|}{$\beta^{*}, \%$} & \multicolumn{2}{c|}{$\gamma, \%$} \\
\cline { 2 - 10 } & by volume & by weight & by volume & by weight & by volume & by weight & by volume & by weight \\
\hline I & 71.01 & 37.40 & 11.87 & 5.66 & 66.39 & 35.10 & 56.11 & 30.37 \\
\hline II & 74.18 & 43.03 & 13.59 & 6.30 & 69.56 & 40.73 & 57.79 & 35.01 \\
\hline III & 78.20 & 59.18 & 12.62 & 5.84 & 74.58 & 56.88 & 63.60 & 51.89 \\
\hline
\end{tabular}

their natural dispersion using expressions 4-6. In the case of Nagornoye oil, the dispersion effectiveness was calculated for the dosing of oil both "by volume" and "by weight".

As can be seen from the data in Table 4, even if the invalid data for dosing Nagornoye oil "by volume" are excluded from the $\beta$ sample, the standard deviation exceeds $10 \%$ and varies from $11.87 \%$ to $13.59 \%$. Moreover, in the case of dosing of oil "by weight", the standard deviation is much lower and varies from $5.66 \%$ to $6.30 \%$. "Std. Dev." refers to the standard deviation of the seven replicates for oil-dispersant systems. Final $\beta^{*}$ was the lower 95\% confidence limit for the nominally dispersed oil, which considered naturally dispersed oil, across seven replicates $(\gamma)$.

The final results on the estimation of the effectiveness of the dispersing ability of dispersants I-III in relation to Usinsk and Nagornoye oils are presented in Table 5.

Table 5. The efficiencies of oil dispersions of the Usinsk and Nagornoye oils with dispersants I-III $(n=7)$ at $P=0.95$

\begin{tabular}{|c|c|c|c|}
\hline \multirow{2}{*}{ Dispersant } & \multicolumn{3}{|c|}{$\beta^{*}, \%$} \\
\cline { 2 - 4 } & \multirow{2}{*}{$\begin{array}{c}\text { Usinsk oil } \\
\text { "by volume" }\end{array}$} & \multicolumn{2}{|c|}{ Nagornoye oil } \\
\cline { 3 - 4 } & & "by volume" & "by weight" \\
\hline I & $82 \pm 8$ & $66 \pm 11$ & $35 \pm 5$ \\
\hline II & $83 \pm 6$ & $70 \pm 12$ & $41 \pm 5$ \\
\hline III & $93 \pm 7$ & $74 \pm 11$ & $56 \pm 5$ \\
\hline
\end{tabular}

As can be seen from the Table 5, the introduction of Nagornoye oil into the tested systems "by weight" is more preferable since this allows minimizing the error of the experiment. It should be noted that the introduction of Nagornoye oil "by weight" also leads to a decrease in the values of the efficiency of the dispersing ability of the tested dispersants. It is known that one of the criteria for the active use of a dispersant is to achieve an efficiency indicator of its dispersing ability of at least 45\% (National regulations, 1996). In this regard, only dispersant III can be considered as a potential dispersant of oil films for heavy oils.With respect to light Usinsk oil, dispersants are more effective than for heavy oil, which is consistent with published data (De Percin et al., 2005; Fingas \& Fieldhouse, 2003; Venosa et al., 2002).

\section{Conclusions}

Currently, BFT has a much higher reproducibility of results compared to other widely used methods (for example, ExDET, WSL) and shows high convergence with the results of field tests of an accidental spill and its response. However there are a sufficient number of publications indicating high errors of the method when assessing the efficiency of dispersion of highly viscous oils. Currently, there is a limited number of publications on the development and analysis of approaches to reduce such errors. The aim of our study was to find approaches to reduce the error of the BFT method when assessing the effectiveness of dispersants for heavy oils. In the course of our research it was shown that one of the approaches to minimizing errors is the introduction of heavy oil into the tested systems by the BFT method not "by volume", as prescribed by standard technique, but "by weight". Experiments to determine the efficiency of dispersion of Nagornoye heavy oil showed a significant variation in the values in the case of adding oil to the system "by volume". This fact, probably, is due to the discrepancy between the actual oil content in the system and the calculated one. Due to the high viscosity of the oil, the introduced volume does not reflect the final oil content in the system, which introduced errors in the calculations of oil dispersion effectiveness. Introduction of Nagornoye oil into the tested systems "by weight" is more preferable since this allows minimizing the error of the experiment. The effectiveness of three dispersants with respect to dispersion of oil from the Usinsk and Nagornoye fields was determined by the BFT method also. It was established that the studied dispersant samples have a higher dispersing ability with respect to the less dense and viscous oil of the Usinsk field.

\section{Acknowledgements}

This research did not receive any specific grant from funding agencies in the public, commercial, or not-for-profit sectors.

\section{Conflict of interest}

The authors declare no conflict of interest. 


\section{References}

Becker, K. W., Coker, L. G., \& Walsh, M. A. (1991). A method for evaluating oil spill dispersants Exxon dispersant effectiveness test (EXDET). OCEANS 91 Proceedings, 23, 1486-1490. https://doi.org/10.1109/OCEANS.1991.606514

Bocard, C., \& Castaing, G. (1986). Dispersant effectiveness evaluation in a dynamic flow-through system: The IFP dilution test. Oil and Chemical Pollution, 3(6), 433-444. https://doi.org/10.1016/S0269-8579(86)80024-7

Brandvik, P. J., Johansen, Ø., Leirvik, F., Farooq, U., \& Daling, P. S. (2013). Droplet breakup in subsurface oil releases Part 1: Experimental study of droplet breakup and effectiveness of dispersant injection. Marine Pollution Bulletin, 73(1), 319-326. https://doi.org/10.1016/j.marpolbul.2013.05.020

Cai, Z., Fu, J., Liu, W., Fu, K., O’Reilly, S. E., \& Zhao, D. (2017). Effects of oil dispersants on settling of marine sediment particles and particle-facilitated distribution and transport of oil components. Marine Pollution Bulletin, 114(1), 408-418. https://doi.org/10.1016/j.marpolbul.2016.09.057

Canevari, G. P., Calcavecchio, P., Becker, K. W., Lessard, R. R., \& Fiocco, R. J. (2001). Key parameters affecting the dispersion of viscous oil. International Oil Spill Conference Proceedings, 2001, 479-483. https://doi.org/10.7901/2169-3358-2001-1-479

Daling, P. S., \& Lichtenthaler, R. G. (1986). Chemical dispersion of oil. Comparison of the effectiveness results obtained in laboratory and small-scale field tests. Oil and Chemical Pollution, 3(1), 19-35. https://doi.org/10.1016/S0269-8579(86)80011-9

De Percin, P., Suidan, M. T., \& Sorial, G. A. (2005). Analysis of dispersant effectiveness of heavy fuel oils and weathered crude pils at two different temperatures using the baffled flask (Report No. 69-C-00-159). National Risk Management Research Laboratory, US Environmental Protection Agency.

Fingas, M., \& Fieldhouse, B. (2003). Studies of the formation process of water-in-oil emulsions. Marine Pollution Bulletin, 47(9-12), 369-396. https://doi.org/10.1016/S0025-326X(03)00212-1

Fingas, M. F., Dufort, V. M., Hughes, K. A., Bobra, M. A., \& Duggan, L. V. (1989). Laboratory studies on oil spill dispersants. In L. Flaherty (Ed.), Oil dispersants: New ecological approaches (pp. 207-219). ASTM International.

Fiocco, R. J., Daling, P. S., DeMarco, G., Lessard, R. R., \& Canevari, G. P. (1999). Chemical dispersibility study of heavy bunker fuel oil. In Proceedings of the $22^{\text {nd }}$ Arctic and Marine Oilspill Program Technical Seminar (Vol. 1, pp. 173-186). Ministry of Supply and Services, Canada.

Holder, E. L., Conmy, R. N., \& Venosa, A. D. (2015). Comparative laboratory-scale testing of dispersant effectiveness of 23 crude oils using four different testing protocols. Journal of Environmental Protection, 6(6), 628-639.

https://doi.org/10.4236/jep.2015.66057

Lee, M., Martinelli, F., Lynch, B,. \& Morris, P. R. (1981). The use of dispersants on viscous fuel oils and water in crude oil emulsions. International Oil Spill Conference Proceedings, 1981(1), 31-35. https://doi.org/10.7901/2169-3358-1981-1-31

Lessard, R. R., \& DeMarco, G. (2000). The significance of oil spill dispersants. Spill Science \& Technology Bulletin, 6(1), 59-68. https://doi.org/10.1016/S1353-2561(99)00061-4

Li, Z., Lee, K., Kepkey, P. E., Mikkelsen, O., \& Pottsmith, C. (2011). Monitoring dispersed oil droplet size distribution at the Gulf of Mexico Deepwater Horizon spill site. International Oil Spill Conference Proceedings, 2011(1), abs 377, 15 pp.

Mackay, D., Chau, A., Hossain, K., \& Bobra, M. (1984). Measurement and prediction of the effectiveness of oil spill chemical dispersants. In T. Allen (Ed.), Oil spill chemical dispersants: Research, experience, and recommendations (pp. 38-54). ASTM International.

Mukherjee, B., Turner, J., \& Wrenn, B. A. (2011). Effect of oil composition on chemical dispersion of crude oil. Environmental Engineering Science, 28(7), 497-506. https://doi.org/10.1089/ees.2010.0226

Panetta, P. D., Jerding, J., Podolski, A., Du, H., \& Byrne, R. (2018). Crude oil viscosity research and dispersant effectiveness measurements (Report No. E17PD00017). US Department of the Interior Bureau of Safety and Environmental Enforcement, Sterling, VA.

Salnikov, A. V., Gribov, G. G., \& Korotkaya, M. L. (2017). Patent of the Russian Federation No 2639330 appl. 28.03.2016, publ. 21.12.2017.

Sorial, G. A., Venosa, A. D., Koran, K. M., Holder, E., \& King, D. W. (2004). Oil spill dispersant effectiveness protocol. II: Performance of revised protocol. Journal of Environmental Engineering, 130(10), 1073-1084. https://doi.org/10.1061/(ASCE)0733-9372(2004)130:10(1085)

Trudel, K., Belore, R., Mullin, J.. \& Guarino, A. (2010). Oil viscosity limitation on dispersibility of crude oil under simulated at-sea conditions in a large wave tank. Marine Pollution Bulletin, 60(9), 1606-1614. https://doi.org/10.1016/j.marpolbul.2010.01.010

US Environmental Protection Agency. (1996). Swirling flask dispersant effectiveness test (Pt. 300, App. C, pp. 224-246). National regulations.

Venosa, A. D., King, D. W., \& Sorial, G. A. (2002). The baffled flask test for dispersant effectiveness: A round robin evaluation of reproducibility and repeatability. Spill Science \& Technology Bulletin, 7(5-6), 299-308. https://doi.org/10.1016/S1353-2561(02)00072-5 